\title{
Tomografia computadorizada multidetectores de baixa dose de radiação para avaliação de canino incluso
}

\section{Low-dose multidetector computed tomography for impacted canine evaluation}

\author{
Juliana P. Renz \\ Gustavo N. Dotto* \\ Kaline T. Antunes ${ }^{* * *}$ \\ Gabriela S. Liedke . $^{* * * *}$
}

\section{Resumo}

Objetivo: avaliar caninos inclusos em tomografias computadorizadas multidetectores (TCMD) realizadas no Hospital Universitário de Santa Maria. Materiais e método: foi realizado um estudo observacional transversal com imagens de um banco de dados de TCMD de baixa dose de radiação, solicitadas por indicações clínicas odontológicas. Os exames em formato digital imaging and communications in medicine foram avaliados por um examinador treinado, no software Weasis Medical Viewer. A avaliação dos exames compreendeu a observação das seguintes características: impactação do canino (uni ou bilateral), localização do canino (maxila ou mandíbula), localização da coroa do canino (vestibular ou lingual/palatal), eixo de erupção, espessura do folículo pericoronário, presença de lesões associadas, presença de reabsorção nos dentes adjacentes e presença de agenesias dentárias ou de supranumerários. Os resultados foram avaliados de maneira descritiva, por meio de prevalência dos achados. Resultados: foram encontrados 21 caninos inclusos em 837 tomografias avaliadas $(1,9 \%)$. Foi observado um predomínio de indivíduos do sexo feminino $(71,4 \%)$, e a retenção do canino foi mais frequente na maxila $(71,4 \%)$ e com impactação unilateral (61,9\%). Quatro caninos apresentaram folículo pericoronário, com espessura maior que $2 \mathrm{~mm}$; 19\% dos exames apresentaram reabsorção radicular externa em algum incisivo lateral. Apenas um canino apresentava lesão associada, sugerindo esclerose óssea. Além dos caninos inclusos, foi possível avaliar alterações relacionadas às demais regiões e estruturas dentomaxilofaciais. Conclusão: apesar da amostra limitada de imagens, o presente estudo demonstrou a aplicabilidade clínica da TCMD com protocolo de baixa dose de radiação, para a avaliação dos caninos inclusos e características relacionadas a esses dentes, bem como de dentes e regiões adjacentes.

Palavras-chave: Dente canino. Dente incluso. Dose de radiação. Tomografia computadorizada multidetectores.

\section{Introdução}

Os caninos são elementos indispensáveis para o sistema estomatognático, mantendo forma e função da dentição, oclusão dinâmica, estética do sorriso e harmonia facial. ${ }^{1}$ São os últimos dentes a irromper na arcada dentária e, por esse motivo, os que mais sofrem impactação, depois dos terceiros molares. A etiologia dessa condição é multifatorial, abrangendo fatores gerais e locais. As principais causas sistêmicas são distúrbios endócrinos, fatores hereditários e síndromes com malformações craniofaciais. Já os fatores locais incluem falta de espaço no arco dentário, distúrbios na sequência de erupção dos permanentes, perda prematura do canino decíduo, anquilose, dentes supranumerários, fissura alveolar e presença de cistos e tumores. Quando não diagnosticados e tratados, caninos inclusos podem desenvolver patologias e/ou ocasionar reabsorções dos dentes adjacentes. Dessa forma, é fundamental um diagnóstico precoce, para que se tenha um prognóstico favorável, evitando complicações e permitindo a melhor tomada de decisão clínica. ${ }^{1,2}$

O diagnóstico da condição intraóssea do canino é fundamental para o sucesso terapêutico. A correta localização do canino incluso no rebordo alveolar bem como sua relação com as estruturas e os demais elementos dentários adjacentes possibilitam planejamento cirúrgico adequado, redução de estresse para o profissional e o paciente, menor tempo operatório e prognóstico mais previsível. ${ }^{2}$ Normalmente o diagnóstico desses casos é realizado por meio de 
exames radiográficos. Entretanto, apesar da baixa dose de radiação e da alta disponibilidade das radiografias, a natureza bidimensional desse exame, com consequente sobreposição de estruturas, determina algumas dificuldades para a interpretação da imagem. Assim, muitas vezes é necessário utilizar mais de uma incidência radiográfica, para permitir uma adequada localização do elemento dentário incluso. A técnica de dissociação radiográfica horizontal, conhecida como Técnica de Clark, auxilia na localização do dente incluso, mostrando se ele se localiza por vestibular ou palatino em relação às raízes dos dentes adjacentes. Contudo, o posicionamento dentário e as dificuldades anatômicas podem limitar a obtenção de imagens adequadas. ${ }^{3-5}$

Com o objetivo de superar as dificuldades inerentes ao exame radiográfico, a tomografia computadorizada (TC) vem sendo cada vez mais empregada na Odontologia. Existem dois tipos de TC: a multidetectores (TCMD) e a de feixe cônico (TCFC). A TCMD adquire múltiplas fatias (ou slices) da região examinada, enquanto o paciente se encontra deitado na mesa do tomógrafo. Já na TCFC, o paciente pode estar deitado, em pé ou sentado, e o aparelho realiza apenas uma volta ao redor da cabeça do paciente. Independentemente do tipo da aquisição tomográfica, são obtidas reconstruções multiplanares e tridimensionais, reproduzindo a região sem sobreposição das demais estruturas, em escala real e sem distorções. ${ }^{6-8}$ Sua aplicação clínica, com elevada precisão, direciona-se a praticamente todas as áreas da Odontologia. ${ }^{9}$ Assim, esse exame apresenta vantagens frente às radiografias, podendo alterar o diagnóstico e o plano de tratamento em algumas situações clínicas, como no caso dos caninos inclusos. ${ }^{3}$

Existe uma preocupação constante dos profissionais da saúde com relação à dose de radiação recebida pelos pacientes. O princípio as low as reasonably achievable (tão baixa quanto razoavelmente possível) estabelece, dentro da proteção radiológica, a redução da dose de radiação a que o paciente é submetido durante a realização de um exame. Essa exposição será clinicamente justificável se os benefícios trazidos pelo exame para o diagnóstico e o plano de tratamento do paciente superarem os riscos da exposição à radiação. ${ }^{10} \mathrm{~A}$ TCFC, atualmente, é considerada o exame de referência na Odontologia, principalmente por apresentar menor exposição à radiação, quando comparada ao protocolo tradicional de aquisição da TCMD. ${ }^{11}$

Os hospitais, normalmente, não possuem equipamentos por imagem odontológicos (panorâmicos, intrabucais ou TCFC). No entanto, a TCMD está presente e pode ser integrada à rotina de diagnósticos odontológicos. Como o objetivo da avaliação odontológica são os tecidos duros, foram propostas algumas modificações no protocolo de aquisição deste equipamento, a fim de reduzir a dose de radiação da TCMD sem comprometer o diagnóstico. ${ }^{12,13}$ As- sim, a TCMD com protocolo de baixa dose passa a ser utilizada para a aquisição específica da região facial, produzindo imagens tridimensionais com uma dose de radiação semelhante à de exames radiográficos. ${ }^{14}$

A utilização da TCMD com baixa dose traz diversos benefícios para a avaliação odontológica dos pacientes em hospitais. Entretanto, no momento, não existem estudos que validem a utilização desse exame como rotina para avaliação odontológica. Assim, os objetivos deste trabalho foram avaliar a prevalência de caninos inclusos nos exames tomográficos realizados no Hospital Universitário de Santa Maria (Husm) e observar aspectos como a localização e o desvio no eixo de erupção do canino incluso, a presença e a extensão de reabsorção radicular nos dentes adjacentes e demais alterações relacionadas aos caninos e às estruturas dentomaxilofaciais.

\section{Materiais e método}

Este projeto de pesquisa foi aprovado pelo comitê de ética da instituição (n⿳⺈ ${ }^{-}$CAAE: 81270117.0.0000.5346). Foi realizado um estudo observacional transversal, utilizando o banco de dados de exames tomográficos do Husm, um hospital público e de referência para a região central do estado do Rio Grande do Sul.

As tomografias foram realizadas no período de julho de 2013 a janeiro de 2018, de pacientes internados ou encaminhados de outros serviços públicos de saúde da região. Todos os exames foram realizados após anamnese e avaliação clínica do paciente, sendo constatada necessidade para complementação do diagnóstico e do plano de tratamento. Como critérios de inclusão, fizeram parte do estudo tomografias que possuíam algum canino incluso sem perspectiva imaginológica de erupção normal. Foram excluídos os exames que não apresentaram qualidade de imagem suficiente para avaliação dentária.

Todos os exames foram realizados no tomógrafo Aquilion 64 (Toshiba America Medical Systems, Inc., Tustin, CA, EUA), com protocolo de aquisição de baixa dose $(120 \mathrm{kV}$ e $10 \mathrm{mAs}$, pitch $=0,62$, voxel de $0,6 \mathrm{~mm}$ [ou inferior] e tempo de aquisição de aproximadamente 6 segundos [0,5s para $360^{\circ}$ de rotação]). O campo de visão dos exames é fixo em $14 \mathrm{~cm}$ de largura facial, sendo que a altura abrange o terço médio da face e a mandíbula. As tomografias foram realizadas com o paciente deitado em decúbito dorsal e dentes ocluídos. ${ }^{14}$

Os exames selecionados foram exportados em formato digital imaging and communications in medicine (Dicom) e avaliados no software Weasis Medical Viewer (GitHub - São Franciso, Califórnia, EUA). Um examinador treinado e calibrado, especialista em radiologia e familiarizado com avaliação tomográfica, realizou a seleção e a avaliação dos exames, o que compreendeu a observação das seguintes características: 
- impactação do canino: uni ou bilateral;

- localização do canino: maxila ou mandíbula;

- localização da coroa do canino: vestibular ou lingual/palatal;

- presença de dilaceração radicular do canino;

- eixo de erupção do canino: verticalizado, horizontalizado ou mesioangulado;

- espessura do folículo pericoronário (medido em mm);

- presença de lesões associadas;

- presença de reabsorção no canino (externa ou interna);

- estágio da rizogênese do canino;

- presença de reabsorção nos dentes adjacentes;

- presença de caninos decíduos;

- agenesia de incisivo lateral;

- dentes supranumerários adjacentes ao canino;

- relação do canino com o canal nasopalatino ou o forame mentual.

Além dessas características, outros achados não relacionados ao canino incluso também foram investigados, como lesões e alterações nos dentes, nas estruturas de suporte e nas estruturas anexas maxilofaciais.

Os resultados foram avaliados de maneira descritiva, utilizando os programas SPSS 13.0 (SPSS, Chicago, IL, USA) e Microsoft Office Excel (Microsoft Corp., Redmond, WA, USA), considerando a prevalência dos achados.

\section{Resultados}

Foram avaliadas 837 tomografias, e foram encontrados 21 caninos inclusos em 16 exames (prevalência de $1,9 \%)$. Na maioria dos exames $(71,4 \%)$, o canino apresentava rizogênese completa. Em quatro casos, foi observada a retenção do dente decíduo no arco.

Foi observado um predomínio de indivíduos do sexo feminino $(71,4 \%)$, em relação ao sexo masculino $(28,6 \%)$. A média de idade foi de 25,6 anos (mínima de 11 e máxima de 63). Ainda, a retenção do canino foi mais frequente na maxila $(71,4 \%)$ e com impactação unilateral $(61,9 \%)$. Apenas um exame apresentou os quatro elementos inclusos. Na Tabela 1 , observa-se a prevalência de caninos inclusos, em relação ao elemento dentário e ao sexo do paciente.

Avaliando o eixo de erupção dos caninos inclusos, $42,9 \%$ apresentavam-se verticalizados; $47,6 \%$, mesioangulados; e 9,5\%, horizontalizados (Figura 1). Com relação ao posicionamento da coroa dos caninos inclusos no rebordo alveolar, a maioria apresentava-se em região lingual/palatal $(61,9 \%)$. Foram encontrados três exames (4 dentes) com presença de reabsorção radicular externa nos dentes adjacentes aos caninos inclusos (19\%) (Figura 2a); outros sete casos foram identificados como apresentando relação íntima da coroa do canino com a raiz de algum dente adjacente. Outros achados relacio- nados aos caninos inclusos foram a relação desse dente com estruturas anatômicas (canal nasopalatino ou forame mentual) $(28,6 \%)$ (Figura $2 \mathrm{~b})$ e dilaceração radicular $(28,6 \%)$ (Figura 2c). Não foram observadas reabsorções nos caninos avaliados nem a presença de elementos dentários supranumerários. A Tabela 2 relaciona a localização da coroa do canino no rebordo alveolar com a presença desses achados tomográficos.

Tabela 1 - prevalência de caninos inclusos, considerando o elemento dentário e o sexo do paciente $(n=21)$

\begin{tabular}{c|c|c|c}
\hline Dente & Sexo & Frequência & Total \\
\hline \multirow{2}{*}{13} & Feminino & 5 & 7 \\
& Masculino & 2 & \\
\multirow{3}{*}{33} & Feminino & 7 & 10 \\
& Masculino & 3 & 2 \\
& Feminino & 1 & \\
& Masculino & 1 & 2 \\
\hline
\end{tabular}

Fonte: autores.

Tabela 2 - Relação da localização da coroa do canino no rebordo alveolar com outros achados tomográficos

\begin{tabular}{l|c|c|c|c|c|c}
\hline \multirow{2}{*}{$\begin{array}{c}\text { Coroa do } \\
\text { canino }\end{array}$} & \multicolumn{2}{|c|}{$\begin{array}{c}\text { Reabsorção } \\
\text { nos dentes } \\
\text { adjacentes }\end{array}$} & \multicolumn{2}{c|}{$\begin{array}{c}\text { Relação com } \\
\text { estruturas } \\
\text { anatômicas }\end{array}$} & \multicolumn{2}{c}{$\begin{array}{c}\text { Dilaceração } \\
\text { radicular }\end{array}$} \\
\cline { 2 - 7 } & Não & Sim & Não & Sim & Não & Sim \\
\hline Palatal/Lingual & 12 & 1 & 9 & 4 & 10 & 3 \\
Vestibular & 3 & 0 & 3 & 0 & 2 & 1 \\
Central & 2 & 3 & 3 & 2 & 3 & 2 \\
\hline
\end{tabular}

Fonte: autores.

O folículo pericoronário dos caninos inclusos foi medido no corte tomográfico axial, na sua maior espessura. Quatro elementos dentários apresentaram folículo pericoronário com espessura maior do que $2 \mathrm{~mm}$, e a espessura média encontrada foi de $1,68 \mathrm{~mm}$ (mínima de 0,08 $\mathrm{mm}$ e máxima de $3,7 \mathrm{~mm}$ ) (Figura 3).

$\mathrm{Na}$ amostra avaliada, os casos de agenesia de incisivo lateral estiveram relacionados com a presença de outras alterações. Um paciente apresentava fissura do rebordo alveolar (Figura 4), e outro, uma extensa área de esclerose óssea adjacente ao canino, bem como a presença de um elemento dentário incluso (dente 31) (Figura 5).

Por fim, todos os exames foram avaliados em relação à presença de alterações não relacionadas diretamente aos caninos inclusos. Foram observadas: alterações dentárias, como raízes residuais, lesões periapicais, cárie dentárias, fraturas dentárias, dentes girovertidos, apinhamentos, dentes inclusos, dentes supranumerários; alterações nas estruturas de suporte dos dentes (reabsorção óssea periodontal); e alterações nas estruturas anexas, como espessamento mucoso no interior dos seios maxilares, calcificação parcial do ligamento estilo-hioide e pontos radiopacos em região de tecidos moles cervicais, sugerindo calcificações arteriais (ateroma). 
Figura 1 - Reconstruções panorâmicas da tomografia computadorizada, evidenciando o eixo de erupção do canino: dente 43 - verticalizado (a), dentes 13 e 23 - horizontalizados (b) e dente 23 - mesioangulado (c).

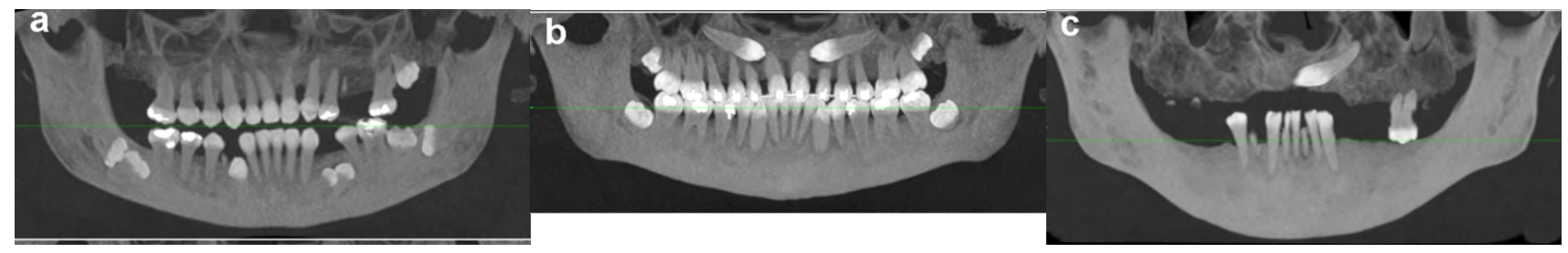

Fonte: autores.

Figura 2 - Cortes multiplanares, exemplificando achados tomográficos relacionados aos caninos inclusos: reabsorção radicular externa de dentes adjacentes (a), relação íntima com o canal nasopalatino (b) e dilaceração radicular (c).

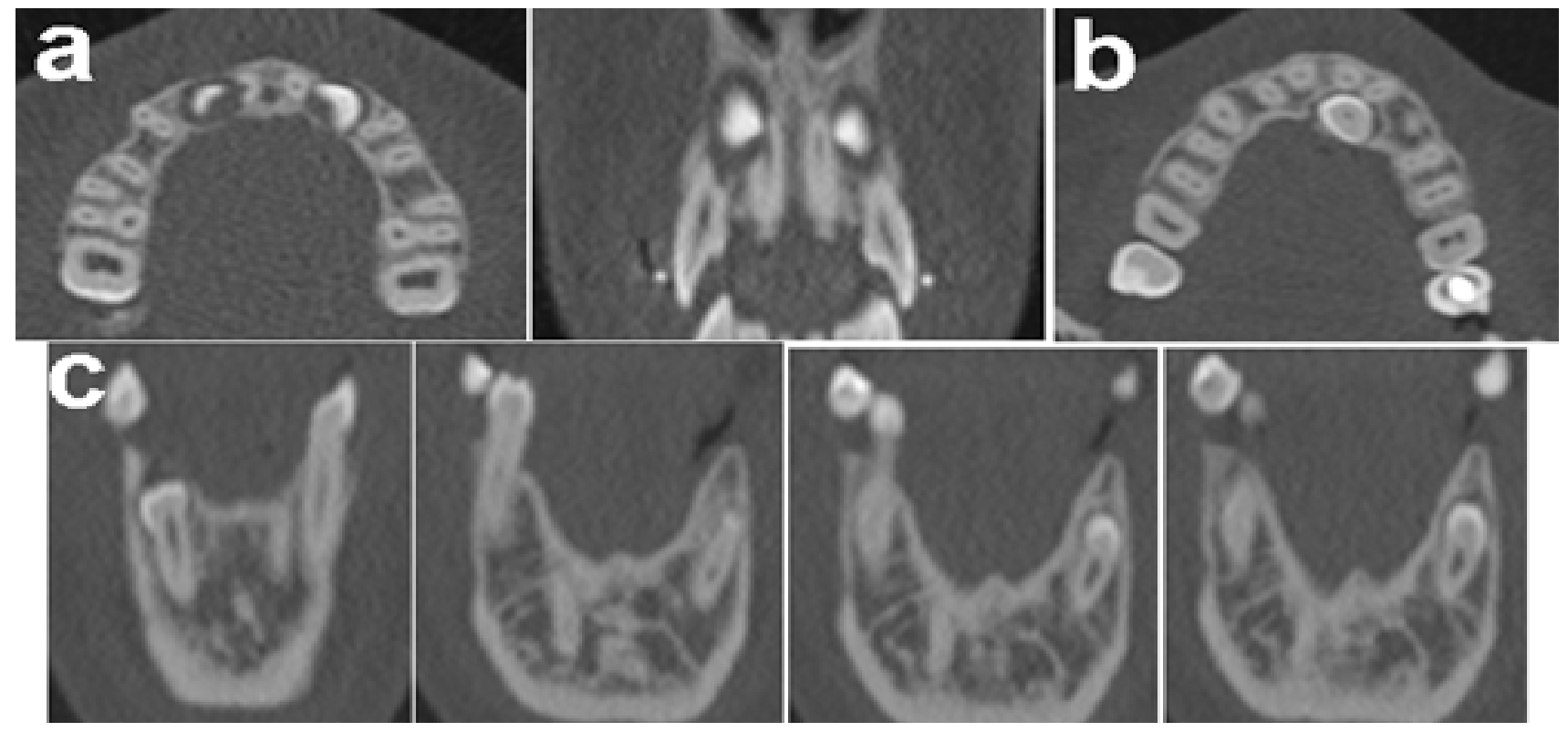

Fonte: autores.

Figura 3 - Cortes axial (a) e coronal (b), evidenciando o folículo pericoronário dos caninos inclusos: dente 13=3,4 mm; e dente $23=3,7 \mathrm{~m}$.
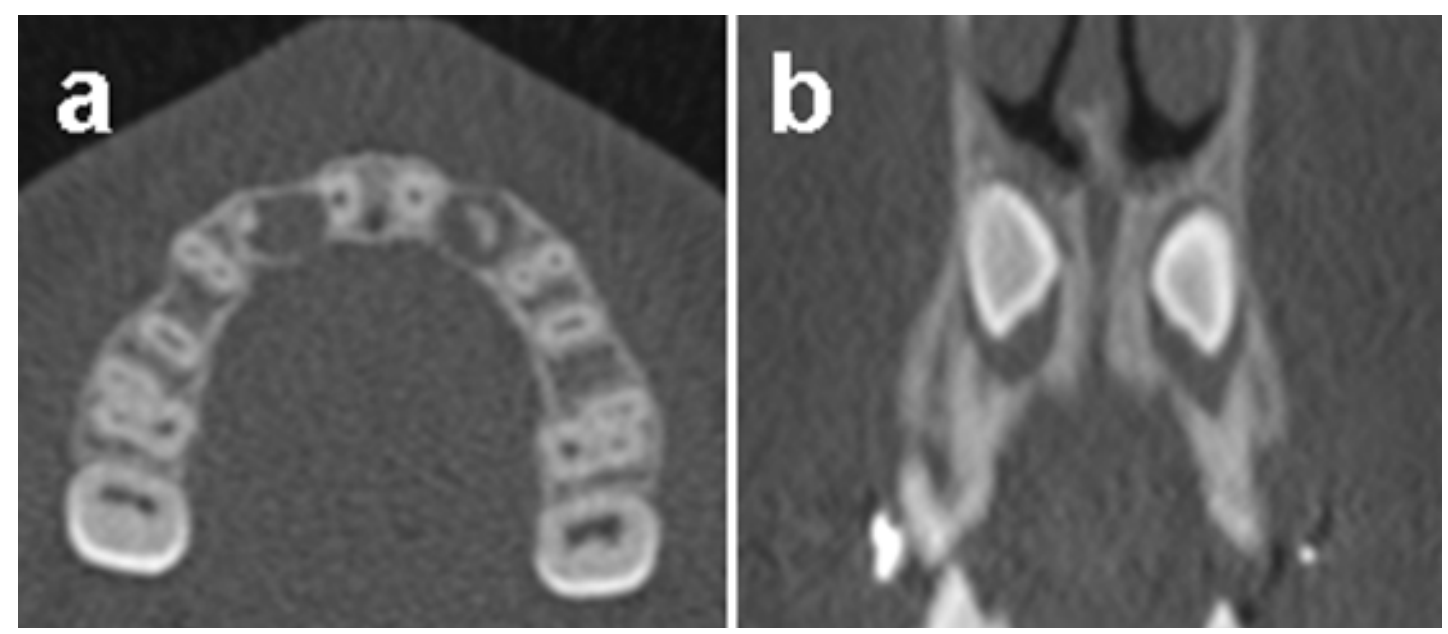

Fonte: autores. 
Figura 4 - Reconstrução panorâmica (a) e corte axial (b), mostrando canino incluso associado à agenesia de incisivo lateral e presença de fissura do rebordo alveolar.
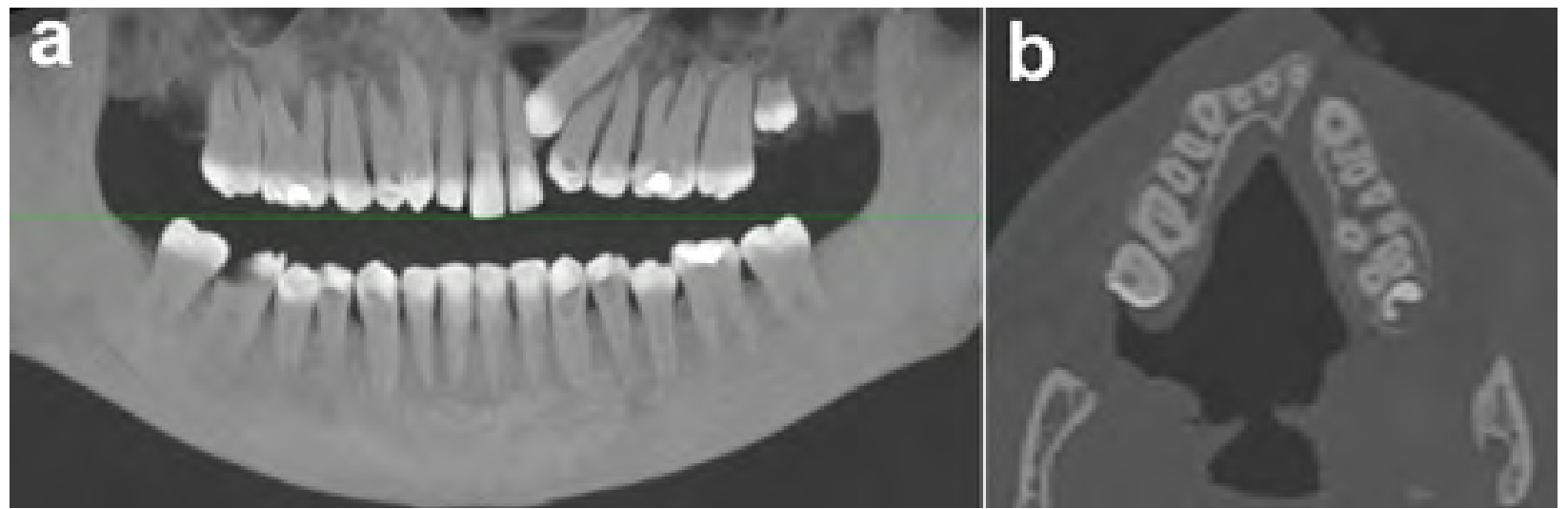

Fonte: autores.

Figura 5 - Reconstrução panorâmica (a) e cortes axiais no nível da coroa e do terço cervical radicular (b), mostrando o dente 33 incluso com lesão de esclerose óssea adjacente.

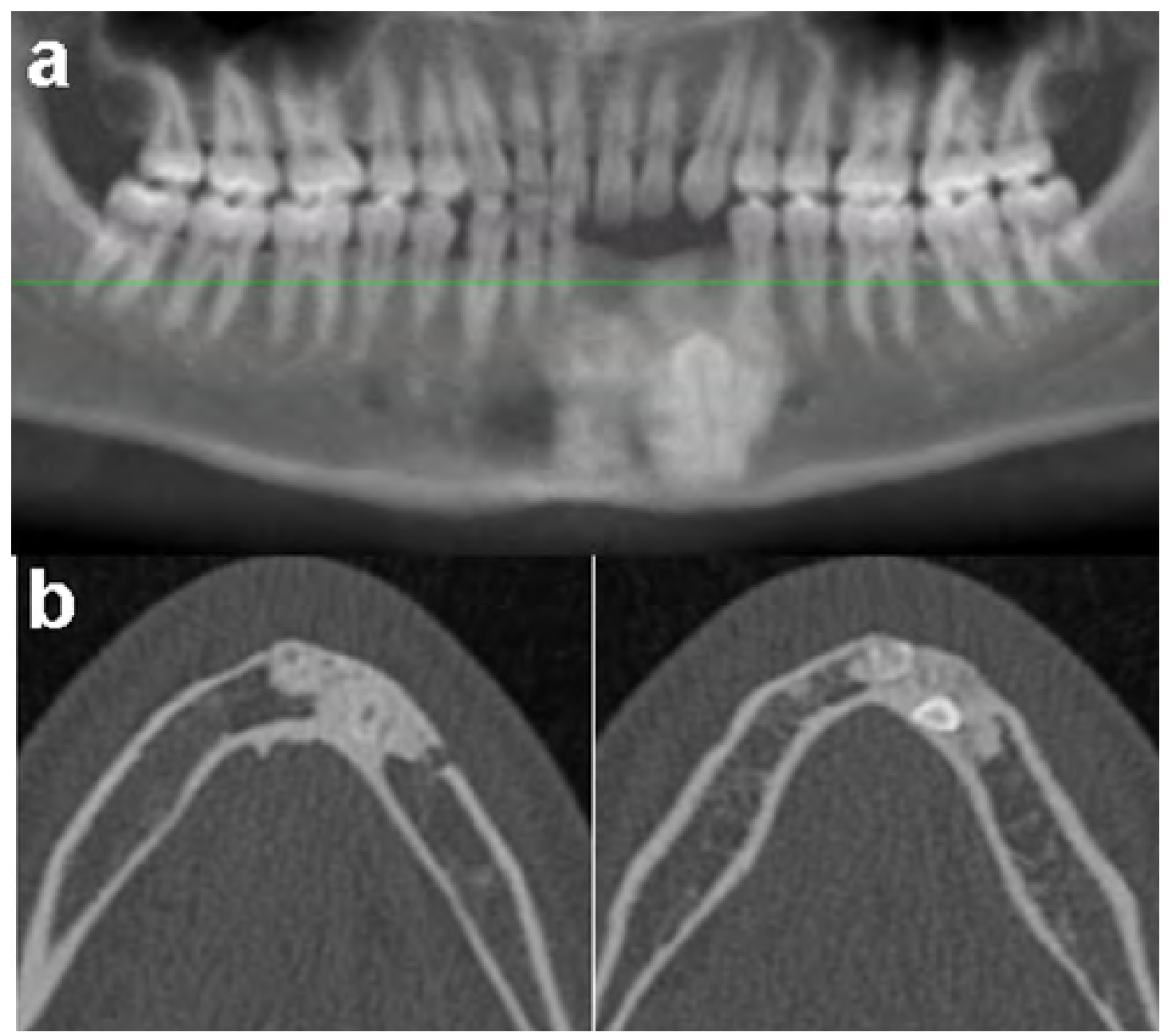

Fonte: autores.

Observação: atentar para a agenesia do dente 32 e a presença do dente 31 incluso, apresentando zonas de reabsorção dentária interna e externa. 


\section{Discussão}

Os caninos são os elementos pilares da arcada dentária, e quanto mais precoce for diagnosticada sua impactação, melhor é o prognóstico do caso. Pela cronologia de erupção, os caninos permanentes devem estar erupcionados na cavidade oral por volta dos 12 anos de idade, porém a erupção tardia de caninos inclusos é comum. ${ }^{15}$ Apesar disso, o posicionamento do canino no arco em idades mais precoces determina sua capacidade de erupção. Por esse motivo, neste estudo, foram selecionados também pacientes mais jovens.

No presente estudo, foi encontrada uma prevalência de $1,9 \%$ de exames com caninos inclusos. Esse dado está de acordo com o estudo de Haney et al. ${ }^{3}$ (2010), em que a incidência variou de $0,8 \%$ a $2,8 \%$, porém é menor do que o observado em outros artigos, que apontaram prevalências de até 3\%. ${ }^{16-19}$ Nesse sentido, devem-se considerar algumas diferenças importantes deste estudo: as imagens avaliadas foram obtidas do banco de imagens de um hospital, de pacientes que realizaram o exame de tomografia para fins não ortodônticos, entre eles, pacientes pré-radioterápicos, especiais e com comprometimento sistêmico, assim justificando a menor prevalência encontrada.

$\mathrm{Na}$ amostra, a maxila teve maior prevalência de caninos inclusos $(71,4 \%)$. O canino superior inicia sua mineralização antes do incisivo e do primeiro molar, tendo o período mais longo e tortuoso de desenvolvimento, e, para finalizar sua irrupção, leva duas vezes mais tempo, tornando-se mais susceptível a alterações na trajetória de erupção. ${ }^{20,21}$ Este estudo também encontrou um predomínio de exames de pacientes do sexo feminino $(71,4 \%)$ e com impactação unilateral (61,9\%), corroborando outras pesquisas que mostraram que a incidência de caninos inclusos é duas vezes maior no sexo feminino, podendo ocorrer de forma uni ou bilateral. ${ }^{22}$

Apesar de as radiografias serem a primeira escolha para a avaliação de pacientes com suspeita de dentes inclusos, esses exames apresentam limitações, como sobreposição de estruturas e ampliação e distorção da imagem. ${ }^{23-25}$ Gavel e Dermaut ${ }^{26}$ (2003) realizaram um estudo avaliando radiografias panorâmicas e cefalométricas, para identificar a posição de dentes inclusos, e concluíram que nunca se deve usar apenas uma imagem radiográfica para o diagnóstico; sugerem, então, a associação de incidências, para um diagnóstico mais preciso. Em contrapartida, a TC é um excelente método para o diagnóstico de elementos dentários inclusos, pois permite visualizar com precisão o dente incluso, em escala real, bem como sua relação com as estruturas adjacentes. Este estudo avaliou imagens provenientes de um novo protocolo de aquisição da TCMD, especialmente desenvolvido para avaliação odontológica de pacientes em hospitais.
Existem algumas diferenças entre os exames de TCMD e de TCFC. Com relação aos parâmetros de exposição, a TCMD possui quilovoltagem $(\mathrm{kVp})$ máxima de $140 \mathrm{kVp}$ e miliamperagem $(\mathrm{mA})$ que pode alcançar até $400 \mathrm{~mA}$; já os parâmetros elétricos de exposição da TCFC variam entre 80 e $120 \mathrm{kVp}$ e entre 3 e $20 \mathrm{~mA}$. Consequentemente, a dose da TCFC é inferior, sendo a primeira escolha para avaliação odontológica. ${ }^{11,27}$ Ao contrário, quando é necessária a avaliação de tecidos moles, a TCMD passa a ser a primeira escolha. ${ }^{11} \mathrm{~A}$ TCMD com baixa dose de radiação, desenvolvida no Husm, mostrou-se uma excelente ferramenta para a avaliação odontológica e, também, de caninos inclusos. $\mathrm{O}$ protocolo apresenta uma dose de radiação 20 vezes menor do que a TCMD tradicional, com tempo de aquisição curto (6 segundos para pacientes adultos e apenas 3 segundos para pacientes infantis), sem comprometer a resolução para avaliação dos tecidos duros. ${ }^{14} \mathrm{~A}$ redução da dose foi alcançada a partir da redução da miliamperagem do exame, conforme proposto por alguns grupos de pesquisa. ${ }^{12,13,28}$ Esses estudos, avaliando exames adquiridos com diferentes miliamperagens (variando de $100 \mathrm{~mA}$ a $10 \mathrm{~mA}$ ) e mantendo o equipamento fixo em $120 \mathrm{kVp}$, verificaram que a resolução espacial se manteve adequada para avaliação dentomaxilofacial. Assim, novos protocolos de aquisição alcançaram redução de até $90 \%$ da dose de radiação e foram denominados de "baixa dose" ou "ultrabaixa dose", quando comparados com o protocolo tradicional da TCMD. Os resultados do presente estudo mostram que, mesmo com a redução expressiva da miliamperagem do exame, foi possível obter imagens de qualidade para avaliação dos caninos inclusos. Ainda, não houve necessidade de deslocamento do paciente para uma clínica odontológica, permitindo um atendimento rápido e eficiente do paciente internado.

A determinação da localização vestibulopalatina do canino incluso e sua relação com as raízes dos dentes adjacentes são fundamentais para o planejamento clínico. Um estudo, comparando imagens radiográficas e tomográficas, concluiu que a TCFC foi o método mais confiável e preciso para diagnóstico da localização de caninos superiores inclusos, bem como da presença de reabsorção radicular nos dentes adjacentes. ${ }^{5}$ Neste estudo, encontrou-se em $61,9 \%$ dos casos a coroa do canino localizada por palatina; em $14,3 \%$, por vestibular; e em $23,8 \%$, a coroa apresentava-se centralizada no rebordo alveolar, corroborando com outros estudos que encontraram de $80 \%$ a $90 \%$ dos caninos inclusos por palatina. ${ }^{27,29,30}$

A reabsorção dos dentes adjacentes pela impactação dos caninos tem sido relatada como resultado do efeito físico do contato entre o canino incluso e o dente adjacente, devido à pressão ativa durante a erupção. ${ }^{31}$ Porém, essa reabsorção pode também ocorrer quando o canino erupcionar normalmente. ${ }^{32}$ A TC permite avaliar com mais precisão a presença 
de reabsorção na raiz dos incisivos adjacentes, em comparação com exames radiográficos. ${ }^{27}$ Estudos relataram que aproximadamente $12 \%$ dos incisivos são reabsorvidos pelos caninos ectópicos, sendo a prevalência quatro vezes maior em meninas do que em meninos. ${ }^{19,33,34}$ Neste estudo, ainda $19 \%$ dos exames apresentou reabsorção radicular externa em algum incisivo lateral.

A presença de lesões adjacentes a dentes inclusos é um achado relativamente frequente, principalmente àqueles que estão por muito tempo nessa condição. Além disso, outras alterações, como odontomas e dentes supranumerários, podem favorecer a rentenção dos caninos. ${ }^{33,35} \mathrm{Na}$ amostra avaliada, apenas um canino apresentava lesão associada, compatível com esclerose óssea.

Geralmente, caninos inclusos apresentam um folículo pericoronário bem desenvolvido, que é visualizado em exames radiográficos como uma faixa radiolúcida ao redor da coroa dental. Esse folículo tem o potencial de originar cistos ou tumores odontogênicos, como é o caso de cisto dentígero, cisto odontogênico calcificante, tumor odontogênico adenomatoide, fibroma ameloblástico ou ameloblastoma, visto que essas lesões são facilmente encontradas relacionadas com dentes inclusos. ${ }^{20,36,37}$ Assim, se não forem diagnosticados e tratados, caninos inclusos podem predispor a presença de patologias ou causar alterações no desenvolvimento oclusal. ${ }^{1} \mathrm{~A}$ maior espessura de folículo pericoronário encontrada no presente estudo foi de $3,7 \mathrm{~mm}$, o que é considerado normal, segundo Consolaro ${ }^{38}$ (1987). O autor realizou medições de espaços pericoronários nas radiografias periapicais e panorâmicas e percebeu que a espessura do folículo pericoronário pode chegar a até 5,6 $\mathrm{mm}$ e, ainda assim, apresentar estrutura e organização normais.

Assim, no presente estudo, a TC agregou valiosas informações clínicas, além do diagnóstico do canino impactado propriamente dito. Esse exame pode ser usado para identificação da morfologia e do comprimento radicular, presença de dilaceração radicular, identificação de inclinações vestibulopalatais, presença de reabsorção radicular externa nos dentes adjacentes e presença de lesões associadas. Com a TC, o ortodontista pode ter informações diagnósticas que não poderiam ser obtidas por exames radiográficos, influenciando diretamente a tomada de decisão clínica. ${ }^{5} \mathrm{O}$ tratamento de caninos inclusos depende de cada caso. Se o paciente optar pelo não tratamento, o acompanhamento longitudinal deve ser criteriosamente realizado. Como opções de tratamento, há as possibilidades de exposição cirúrgica do canino, permitindo sua erupção, exposição cirúrgica e tracionamento ortodôntico, extração do canino e reposicionamento dos dentes adjacentes, extração do canino e reestabelecimento da oclusão por prótese e/ou implante. ${ }^{1}$

Os equipamentos de TCMD estão em constante evolução. Assim, a utilização de um modelo de
64 canais, atualmente em substituição por modelos com 128 ou 256 detectores, poderia ser considerada uma limitação para o estudo. Entretanto, tratando-se de um hospital público, este equipamento ainda proporciona a aquisição das imagens de modo satisfatório.

A Comissão Europeia para Proteção Radiológica e a Academia Europeia de Radiologia Dentomaxilofacial, por meio de um grupo formado por dezenas de pesquisadores e instituições de ensino internacionais, formularam o projeto Sedentexct (2012), ${ }^{11}$ que apresenta um guia para a utilização da TFCF na Odontologia. Entre as recomendações, destaca-se que um exame tomográfico só deverá ser solicitado após extensa avaliação clínica, considerando que as imagens venham a agregar informações novas ao diagnóstico e ao planejamento do caso, devendo-se evitar aquisições rotineiras. Assim, quando bem indicada, a TCFC seria apropriada, uma vez que apresenta menor dose de radiação em comparação à TCMD. Entretanto, considerando as pesquisas mais recentes realizadas com a TCMD com baixa dose de radiação $0^{12-14,28}$ e os resultados deste estudo, sugere-se que este protocolo também possa ser utilizado, caso um equipamento de TCFC não esteja disponível.

O cirurgião-dentista busca conhecer o estado de saúde geral do paciente ${ }^{39}$ e colaborar com o seu bem-estar, sendo responsável pela identificação de alterações no complexo bucomaxilofacial. ${ }^{40}$ Além das alterações relacionadas aos caninos inclusos, puderam ser avaliadas outras mudanças nas imagens avaliadas que não estavam relacionadas aos caninos. Entre elas, um exame apresentava imagem compatível com placa de ateroma, que é a manifestação da aterosclerose, doença inflamatória crônica e progressiva que acomete artérias de calibre grande e intermediário, podendo gerar obstrução arterial e acidente vascular cerebral no caso da artéria carótida. ${ }^{41}$ Além desse achado, também foram identificadas alterações dentárias, periapicais e periodontais, reiterando que sempre que um exame tomográfico é realizado, ele deve ser investigado em toda sua extensão, contemplando outros diagnósticos além daquele que levou à sua solicitação inicial. ${ }^{11}$

\section{Conclusão}

Este estudo, apesar da amostra limitada de imagens, demonstrou a aplicabilidade clínica da TCMD com protocolo de baixa dose de radiação para a avaliação dos caninos inclusos e características relacionadas a esses dentes, bem como dos dentes e regiões adjacentes. A utilização deste protocolo para avaliação odontológica de pacientes internados e ambulatoriais deveria ser estimulada e ampliada para os demais hospitais que também possuem equipamento de TCMD. 


\section{Agradecimentos}

Os autores agradecem a Unidade e-Saúde do Hospital Universitário de Santa Maria pela colaboração para a realização desta pesquisa.

\section{Abstract}

Objective: to evaluate impacted canines by tomographic exams performed at the University Hospital of Santa Maria. Materials and method: a cross-sectional observational study was performed with selected images from a database of low dose computed tomography $(\mathrm{CT})$ scans requested by several dental indications. The DICOM files were evaluated by a trained examiner, using Weasis Medical Viewer software. The evaluation of each exam included the following characteristics: canine impaction (uni or bilateral), location of the canine (maxilla or mandible), location of the canine crown (buccal or lingual / palatal), shaft of canine eruption, thickness of the pericoronal follicle, presence of associated lesions, presence of external root resorption in adjacent teeth and presence of dental agenesis or supernumerary tooth. The results were evaluated in a descriptive way, through prevalence of the findings. Results: a total of 21 impacted canines were found in 837 CT scans evaluated $(1,9 \%)$. A predominance of female patients was observed (71,4\%); canine impaction was more frequent in the maxilla $(71,4 \%)$, and unilateral $(61,9 \%)$. In $19 \%$ of the exams, external root resorption occurred in some lateral incisor. Four dental elements presented pericoronal follicles thicker than $2 \mathrm{~mm}$. In the evaluated sample, only one canine presented an associated lesion, suggesting bone sclerosis. Beyond the canine-related, other dental and maxillofacial changes were also evaluated. Conclusion: in spite of the limited sample, the present study demonstrated the clinical applicability of low dose CT for the evaluation of impacted canines, and characteristics related to these teeth, as well as to the adjacent teeth and regions.

Keywords: Cuspid. Tooth impacted. Radiation dosage. Multidetector computed tomography.

\section{Referências}

1. Cappellette M, Cappellette Júnior M, Fernandes LCM, Oliveira AP, Yamamoto LH, Shido FT, et al. Caninos permanentes inclusos por palatino: diagnóstico e terapêutica - uma sugestão técnica de tratamento. Dental Press Ortodontia e Ortopedia Facial 2008; 13(1):60-73.

2. Primo BT, Andrade MGS, Oliveira HW, Oliveira MG. Dentes inclusos: novas perspectivas de localização. Ver Fac Odontol Univ Passo Fundo 2011; 16(1):95-9.

3. Haney E, Gansky SA, Lee JS, Johnson E, Maki K, Miller AJ, et al. Comparative analysis of traditional radiographs and cone-beam computed tomography volumetric images in the diagnosis and treatment planning of maxillary impacted canines. Am J Orthodontics and Dentofacial Orthopedics 2010; 137(5):590-7.

4. Nogueira AS, Santana GB, Bezerra TP. Diagnóstico por imagens das inclusões dentárias. In: Nogueira AS. Abordagem contemporânea dos dentes inclusos. 1. ed. São Paulo: Santos; 2004. p. 11-29.
5. Tsolakis AI, Kalavritinos M, Bitsanis E, Sanoudos M, Benetou V, Alexiou K, et al. Reliability of different radiographic methods for the localization of displaced maxillary canines. Am J Orthodontics and Dentofacial Orthopedics 2018; 153(2):308-14

6. Cavalcanti, M. Tomografia Computadorizada por Feixe Cônico: Interpretação e Diagnóstico para o Cirurgião dentista. 1. ed. São Paulo: Livraria Santos Ltda; 2010.

7. Garib DG, Júnior RR, Raymundo MV, Raymundo DV, Ferreira SN. Tomografia computadorizada de feixe cônico (Cone beam): entendendo este novo método de diagnóstico por imagem com promissora aplicabilidade na Ortodontia. Dental Press Ortodontia e Ortopedia Facial 2007; 12(2):139-56.

8. Quereshy FA, Savell TA, Palomo JM. Applications of cone beam computed tomography in the practice of oral and maxillofacial surgery. J Oral and Maxillofacial Surgery 2008; 66(4):791-6.

9. De Vos W, Casselman J, Swennen GRJ. Cone-beam computerized tomography (CBCT) imaging of the oral and maxillofacial region: a systematic review of the literature. Int J Oral Maxillofac Surg 2009; 38:609-25.

10. Scarfe WC, Farman AG. What is cone beam CT and how does it work? Dent Clin N Am 2008; 52:707-30.

11. Sedentex CT Project. Radiation protection $\mathrm{n} \square$ 172: Cone Beam CT for dental and maxillofacial radiology. Luxembourg: European Commission Directorate-General for Energy; 2012.

12. Almashraqi AA, Ahmed EA, Mohamed NS, Barngkgei IH, Elsherbini NA, Halboub ES. Evaluation of different low-dose multidetector CT and cone beam CT protocols in maxillary sinus imaging: part I-an in vitro study. Dentomaxillofac Radiol 2017; 46(6):20160323.

13. Widmann G, Bischel A, Stratis A, Bosmans H, Jacobs R, Gassner EM, et al. Spatial and contrast resolution of ultralow dose dentomaxillofacial CT imaging using iterative reconstruction technology. Dentomaxillofac Radiol 2017; 46(4):20160452.

14. Dotto PP, Haygert CJP, D'ornellas MC. CTdBem - Tomografia Multislice de baixa dose de radiação. Santa Maria: $\mathrm{pE}$. Editoria Experimental, Universidade Federal de Santa Maria; 2015.

15. Iramaneerat S, Cunningham SJ, Horrocks EN. The effect of two alternative methods of canine exposure upon subsequent duration of orthodontic treatment. Int $J$ Paediatric Dentistry 1998; 8(2):123-9.

16. Al-nimri K, Gharaibeh T. Space conditions and dental and occlusal features in patients with palatally impacted maxillary canines: an aetiological study. Eur J Orthodontics 2005; 27(5):461-5.

17. Garib DG, Henriques JFC, Freitas MR, Janson GRP. Caninos superiores inclusos: preceitos clínicos e radiográficos. Rev Dental Press Ortodontia e Ortopedia Maxilar 1999; 4(4):14-20.

18. Tanaka O, Daniel RF, Vieira SW. O dilema dos caninos superiores inclusos. Rev Ortod Gaúcha 2000; 4(2):122-8.

19. Turner JP, Bedi R. Combined orthodontic and restorative management of a case of bilateral ectopic canines and resorbed central incisors. Br Dent J 1996; 180(2):67-72.

20. Almeida RR, Fuziy A, Almeida MR, Almeida-Pedrin RR, Henriques JFC, Insabralde CMB. Abordagem da impactação e/ou irrupção ectópica dos caninos permanentes: considerações gerais, diagnóstico e terapêutica. Rev Dental Press de Ortodontia e Ortopedia Facial 2001; 6(1)93-116. 
21. Ericson S, Kurol J. Radiographic assessment of maxillary canine eruption in children with clinical signs of eruption disturbance. Eur J Orthodontics 1986; 8:133-40.

22. Becker A, Smith P, Behar R. The incidence of anomalous maxillary lateral incisors in relation to palatally-displaced cuspids. Angle Orthod 1981; 51:24-9.

23. Gratt BM. Panoramic radiography. In: Goaz P, White S, editors. Oral radiology: principles and interpretation. 3. ed. St Louis: CV Mosby; 1994. p. 242-4.

24. Oliveira FAM, Oliveira MG, Oliveira HW, Abreu AT. O uso da tomografia computadorizada na localização de caninos permanentes superiores. Rev Odonto Ciência 2000; 15(30):717.

25. Wriedt S, Jaklin J, Al-Nawas B, Wehrebein H. Impacted upper canines: examination and treatment proposal based on 3D versus 2D diagnosis. J Orofacial Orthopedics 2012; 73:28-40.

26. Gavel V, Dermaut LR. The effect of changes in tooth position of unerupted canines on cephalogramas. Eur J Orthodontics 2003; 25(1):49-56.

27. Schmuth GPF, Freisfeld M, Köster O, Schüller H. The application of computerized tomography (CT) in cases of impacted maxillary canines. Eur J Orthodontics 1992; 14(4):296-301.

28. Haygert CJP, D’Ornellas MC, Welfer D, Bastos RM, Dotto GN. CTdBem - A New Protocol for Ultra Low Radiation Dose MDCT. Stud Health Technol Inform 2017; 245:1029-32.

29. Filho LC, Consolaro A, Cardoso MA, Siqueira DF. Perfuração do esmalte para o tracionamento de caninos: vantagens, desvantagens, descrição da técnica cirúrgica e biomecânica. Dental Press J Orthod 2011; 16(5):172-205.

30. Valdrighi HC, Young AAA, Coser RM, Chiavini PCR. Métodos para tracionamento de caninos inclusos. Ver Gaúcha Odontologia 2004; 52(3):219-22.

31. Ericson S, Bjerklin K, Falahat B. Does the Canine Dental Follicle Cause Resorption of Permanent Incisor Roots? A Computed Tomographic Study of Erupting Maxillary Canines. Angle Orthod 2002; 72(2):95-104.

32. Stewart JA, Heo G, Glover KE, Williamson PC, Lam EWN, Major PW. Factors that relate the treatment duration for patients with palatally impacted maxillary canines. Am J Orthodontics and Dentofacial Orthopedics 2001; 119(3):216-25.

33. Bishara SE. Impacted maxillary canines: a review. Am J Orthodontics and Dentofacial Orthopedics 1992; 101(2):159-70.

34. Ericson S, Kurol J. Incisor resorption caused by maxillary cuspids. A radiographic study. Angle Orthod 1987; 57:332-46.

35. Vishnoi P, Keshubhai KJ, Surendra SS, Bandi N, Jingar J, Rutvik T. Maxillary Canine Impactions: Orthodontic and Surgical Management. Annals Int Med Dental Research 2016; 2(3):2-10.

36. Lewis PD. Preorthodontic surgery in the treatment of impacted canines. Am J Orthod 1971; 60(4):382-97.

37. Mason C, Papadakou P, Roberts GJ. The radiographic localization of impacted maxillary canines: a comparison of methods. Eur J Orthodontics 2001; 23(1):25-34.

38. Consolaro A. Caracterização microscópica de folículos pericoronários de dentes não irrompidos e parcialmente irrompidos. Sua relação com a idade. [Tese de Doutorado]. Bauru (SP): Universidade de São Paulo; 1987.

39. Romiti CBB. Análise da ocorrência de imagens sugestivas de calcificações da artéria carótida em radiografias panorâmicas. Mato Grosso do Sul. [Dissertação de Mestrado em Saúde e Desenvolvimento] Campo Grande: Faculdade de Medicina da Universidade Federal de Mato Grosso do Sul; 2009.
40. Oppermann RV, Rosing CK. Periodontia - Ciência e Clínica. São Paulo: Artes Médicas; 2001.

41. Carvalho ACA, Oliveira LSAF, Melo DP, Crusoé-Rebello I, Campos PSF. Desenvolvimento de placas de ateroma em pacientes diabéticos e hipertensos. Rev de Ciênc Médicas e Biológicas 2010; 9(1):73-7.

\section{Endereço para correspondência:}

Kaline T. Antunes

Programa de Pós-Graduação em Ciências Odontológicas

Rua Marechal Floriano Peixoto, 1184, prédio da antiga reitoria, sala 312 , Centro

97015-372, Santa Maria, RS, Brasil

Telefone: (+55) (55) 99602-2545

E-mail: kaortodontista@gmail.com

Recebido: 01/09/18. Aceito: 24/10/18. 